\title{
The minimal set of Ingleton inequalities
}

\author{
Laurent Guillé \\ ENST Paris
}

\author{
Terence H. Chan and Alex Grant \\ Institute for Telecommunications Research \\ University of South Australia
}

\begin{abstract}
The Ingleton-LP bound is an outer bound for the multicast capacity region, assuming the use of linear network codes. Computation of the bound is performed on a polyhedral cone obtained by taking the intersection of half-spaces induced by the basic (Shannon-type) inequalities and Ingleton inequalities. This paper simplifies the characterization of this cone, by obtaining the unique minimal set of Ingleton inequalities. As a result, the effort required for computation of the Ingleton-LP bound can be greatly reduced.
\end{abstract}

\section{INTRODUCTION}

The network coding approach introduced in [1], [2] generalizes routing by allowing intermediate nodes to forward packets that are coded combinations of all received data packets. One fundamental problem in network coding is to understand the capacity region and the classes of codes that achieve capacity.

In the single session multicast scenario, the problem is well understood - the capacity region is characterized by maxflow/min-cut bounds and linear network codes are sufficient to achieve maximal throughput [2], [3]. Significant practical and theoretical complications arise in more general multicast scenarios, involving more than one session.

There are only a few tools in the literature for study of the capacity region. One powerful theoretical tool bounds the capacity region by the intersection of a set of hyperplanes (specified by the network topology and connection requirement) and the set of entropy functions $\Gamma^{*}$ (inner bound), or its closure $\bar{\Gamma}^{*}$ (outer bound) [4]-[6]. The set of entropy functions is formally introduced in Section II

In fact an exact expression for the capacity region has been obtained, again in terms of $\Gamma^{*}$ [7]. Unfortunately, the capacity region, or even the bounds cannot be computed in practice, due to the lack of an explicit characterization of the set of entropy functions for more than three random variables. One way to resolve this difficulty is via relaxation of the bound, replacing the set of entropy functions with the set of polymatroids $\Gamma$. This yields the linear programming (LP) bound [6].

The LP bound can however be quite loose. While the set of polymatroids $\Gamma^{*}$ is polyhedral, the set of entropy functions $\Gamma$ is not [8]. The addition of any finite number of linear inequalities to the LP bound cannot tighten it to the capacity region. Furthermore, the LP bound holds for any choice of network codes (linear or non-linear). Hence, the LP bound can be even looser when restricted to linear network codes. To address the issue, a modified LP bound was proposed in [9].

The idea of the modified bound is quite simple. Given any network code, the source messages and transmitted link messages are random variables. Restriction to linear codes requires that the corresponding entropy function satisfies the Ingleton inequality. As a result, we can tighten the LP bound for linear network codes by replacing $\Gamma$ (the set of polymatroids) with $\Gamma^{\mathrm{In}}$ (a subset of $\Gamma$ that satisfies all Ingleton inequalities).

Efficiently computation of this Ingleton-LP bound requires a compact and explicit characterization of $\Gamma^{\mathrm{In}}$. As the set $\Gamma^{\mathrm{In}}$ is the intersection of many half-spaces, one can greatly simplify the characterization by identifying which inequalities (or halfspaces) are redundant, meaning that they are implied by other inequalities. The main objective of this paper is to understand the relationship between these half-spaces, so as to simplify the characterization of $\Gamma^{\mathrm{In}}$. Our main result, Theorem 3 in Section [II] is the identification of the unique minimal set of Ingleton inequalities. For reasons of space, all simple proofs have been omitted, and longer proofs are given in sketch form.

\section{ENTROPY FUNCTIONS}

Let $\mathcal{N} \triangleq\{1,2, \cdots, n\}$ induce a $2^{n-1}$-dimensional real Euclidean space $\mathcal{F}_{n}$ whose coordinates are indexed by the set of all nonempty subsets $\alpha \subseteq \mathcal{N}$. Each $h \in \mathcal{F}_{n}$ is defined by $(h(\alpha): \alpha \subseteq \mathcal{N})$. Although $h(\alpha)$ is not defined for the empty set $\emptyset$, we will assume $h(\emptyset)=0$. Points $h \in \mathcal{F}_{n}$ can also be considered as functions $h: 2^{\mathcal{N}} \mapsto \mathbb{R}$.

Definition 1 (Entropic function): A function $h \in \mathcal{F}_{n}$ is entropic if there exists discrete random variables $X_{1}, \ldots, X_{n}$ such that the joint entropy of $\left\{X_{i}: i \in \alpha\right\}$ is $h(\alpha)$ for all $\emptyset \neq \alpha \subseteq N$. Furthermore, $h$ is almost entropic if it is the limit of a sequence of entropic functions.

Let $\Gamma_{n}^{*}$ be the set of all entropic functions. Its closure $\bar{\Gamma}_{n}^{*}$ (i.e., the set of all almost entropic functions) is well-known to be a closed, convex cone [10]. An important recent result with significant implications for $\bar{\Gamma}_{n}^{*}$ is a series of linear information inequalities obtained in [11]. Using this series, $\bar{\Gamma}_{n}^{*}$ was proved to be non-polyhedral for $n \geq 4$. This means that $\bar{\Gamma}_{n}^{*}$ cannot be defined by an intersection of any finite number of linear information inequalities.

To simplify notation, set union will be denoted by concatenation, and singletons and sets with one elements are not distinguished. For any $\alpha, \beta \subseteq \mathcal{N}$ define

$$
\begin{aligned}
h(\alpha \mid \beta) & \triangleq h(\alpha \beta)-h(\beta) \\
I_{h}(\alpha ; \beta \mid \delta) & \triangleq h(\alpha \delta)+h(\beta \delta)-h(\delta)-h(\alpha \beta \delta) .
\end{aligned}
$$

Proposition 1: Let $h \in \bar{\Gamma}_{n}^{*}$. Then for all $\alpha, \beta, \delta \subseteq \mathcal{N}$,

$$
\begin{aligned}
h(\alpha \mid \beta) & \geq 0 \\
I_{h}(\alpha ; \beta \mid \delta) & \geq 0 .
\end{aligned}
$$


Proposition 1 gives the basic inequalities, namely the nonnegativity of (conditional) entropy (1) and of (conditional) mutual information (2). This set of basic inequalities is redundant, and the unique minimal set of basic inequalities that yields all basic inequalities (as linear combinations) is as follows

$$
\begin{aligned}
& h(i \mid \mathcal{N} \backslash i) \geq 0 \\
& I_{h}(i ; j \mid \delta) \geq 0
\end{aligned}
$$

where $i \neq j \in \mathcal{N}$ and $\delta \subseteq \mathcal{N} \backslash\{i, j\}$. Inequalities (3) and (4) are called elemental basic inequalities. See [5] for discussion of basic and elemental inequalities.

For any linear expression $\sum_{\alpha \subseteq \mathcal{N}} c_{\alpha} h(\alpha)$, define the projection onto a subset $\beta$ of $\mathcal{N}$

$$
\sum_{\alpha \subseteq \mathcal{N}} c_{\alpha} h(\beta \cap \alpha)
$$

Similarly, define the projection away from $\beta$

$$
\sum_{\alpha \subseteq \mathcal{N}} c_{\alpha} h(\alpha \backslash \beta)
$$

Clearly, if two linear expressions $\sum_{\alpha \subseteq \mathcal{N}} c_{\alpha} h(\alpha)$ and $\sum_{\alpha \subseteq \mathcal{N}} d_{\alpha} h(\alpha)$ are the same (i.e, the two expressions map to the same real number for all $h \in \mathcal{F}_{n}$ ), then their projections onto (or away from) any subset $\beta$ are identical.

\section{INGLETON INEQUALITIES: \\ PROPERTIES AND THE MINIMAL SET}

Definition 2 (Ingleton Inequality): An Ingleton inequality $J\left(h ; \alpha_{1}, \alpha_{2}, \alpha_{3}, \alpha_{4}\right) \geq 0$ is a linear inequality over $\mathcal{F}_{n}$ defined in terms of four subsets $\alpha_{1}, \alpha_{2}, \alpha_{3}, \alpha_{4}$ of the ground set $\mathcal{N}$ where the Ingleton term $J\left(h ; \alpha_{1}, \alpha_{2}, \alpha_{3}, \alpha_{4}\right)$ is defined as

$$
\begin{aligned}
& h\left(\alpha_{1} \alpha_{2}\right)+h\left(\alpha_{1} \alpha_{3}\right)+h\left(\alpha_{1} \alpha_{4}\right)+h\left(\alpha_{2} \alpha_{3}\right)+h\left(\alpha_{2} \alpha_{4}\right) \\
& \quad-h\left(\alpha_{1}\right)-h\left(\alpha_{2}\right)-h\left(\alpha_{3} \alpha_{4}\right)-h\left(\alpha_{1} \alpha_{2} \alpha_{3}\right)-h\left(\alpha_{1} \alpha_{2} \alpha_{4}\right) .
\end{aligned}
$$

Remark: Originally, the Ingleton inequality refers to the case when $\alpha_{1}, \ldots, \alpha_{4}$ are distinct subsets of singletons. We extend its use to allow arbitrary subsets $\alpha_{1}, \cdots, \alpha_{4}$ of $\mathcal{N}$.

Within the class of Ingleton inequalities indexed by four subsets of $\mathcal{N}$, some are trivial inequalities while some can be derived from basic inequalities or other Ingleton inequalities. For example, if $\alpha_{1}=\alpha_{2}=\alpha_{4}=\alpha_{4}$, then $J\left(h, \alpha_{1}, \ldots, \alpha_{4}\right)=$ 0 for all $h$ and the corresponding Ingleton inequality is trivial.

We now list several properties of the Ingleton term, leading to the minimal set of non-redundant Ingleton inequalities.

Property 1 (Symmetry):

$$
\begin{aligned}
J\left(h ; \alpha_{1}, \alpha_{2}, \alpha_{3}, \alpha_{4}\right) & =J\left(h ; \alpha_{2}, \alpha_{1}, \alpha_{3}, \alpha_{4}\right) \\
& =J\left(h ; \alpha_{1}, \alpha_{2}, \alpha_{3}, \alpha_{4}\right) \\
& =J\left(h ; \alpha_{1}, \alpha_{2}, \alpha_{4}, \alpha_{3}\right) .
\end{aligned}
$$

Thus exchanging $\alpha_{1}$ with $\alpha_{2}$ or $\alpha_{3}$ with $\alpha_{4}$ does not change the value of the Ingleton term, and the number of distinct Ingleton inequalities is at most $2^{4 n-2}$.

Property 2 (Extending basic inequalities):

$$
\begin{aligned}
& J\left(h ; \alpha_{1}, \alpha_{2}, \emptyset, \alpha_{4}\right)=I_{h}\left(\alpha_{1} ; \alpha_{2} \mid \alpha_{4}\right) \\
& J\left(h ; \alpha_{1}, \alpha_{1}, \emptyset, \alpha_{2}\right)=h\left(\alpha_{1} \mid \alpha_{2}\right) .
\end{aligned}
$$

Thus all basic inequalities are special cases of Ingleton inequalities via proper selection of $\alpha_{1}, \ldots, \alpha_{4}$. Hence, $\Gamma_{n}^{\mathrm{In}} \subset \Gamma_{n}$.

Property 3: Let $\alpha_{1}, \ldots, \alpha_{4}, \beta \subseteq \mathcal{N}$. If $\beta \subseteq \alpha_{1} \cap \alpha_{2}$, then

$J\left(h ; \alpha_{1}, \alpha_{2}, \alpha_{3}, \alpha_{4}\right)=J\left(h ; \alpha_{1}, \alpha_{2}, \alpha_{3} \beta, \alpha_{4} \beta\right)+h\left(\beta \mid \alpha_{3}, \alpha_{4}\right)$.

If $\beta \subseteq \alpha_{1} \cap \alpha_{3}$, then we have

$$
\begin{aligned}
& J\left(h ; \alpha_{1}, \alpha_{2}, \alpha_{3}, \alpha_{4}\right) \\
& \quad=J\left(h ; \alpha_{1}, \alpha_{2} \beta, \alpha_{3}, \alpha_{4} \beta\right)+I_{h}\left(\beta ; \alpha_{4} \mid \alpha_{2}\right) .
\end{aligned}
$$

On the other hand, if $\beta \subseteq \alpha_{3} \cap \alpha_{4}$, then

$$
\begin{aligned}
& J\left(h ; \alpha_{1}, \alpha_{2}, \alpha_{3}, \alpha_{4}\right) \\
& \quad=J\left(h ; \alpha_{1} \beta, \alpha_{2} \beta, \alpha_{3}, \alpha_{4}\right)+I_{h}\left(\beta ; \alpha_{2} \mid \alpha_{1}\right)+h\left(\beta \mid \alpha_{2}\right) .
\end{aligned}
$$

To summarize, if an element appears in at least two subsets of an Ingleton term, then we add that element to the remaining two subsets without increasing the value of the Ingleton term.

Property 4: Let $a \subseteq \alpha_{2}, b \subseteq \alpha_{3}$ and $c \subseteq \alpha_{4}$, then

$$
\begin{array}{r}
J\left(h ; a b c, \alpha_{2}, \alpha_{3}, \alpha_{4}\right)=I_{h}\left(\alpha_{3} ; \alpha_{4} \mid a b c\right)+I_{h}\left(\alpha_{3} ; c \mid \alpha_{2} a\right) \\
+I_{h}\left(\alpha_{4} ; b \mid \alpha_{2}\right)+h\left(a \mid \alpha_{3} \alpha_{4}\right) .
\end{array}
$$

Similarly, if $a \subseteq \alpha_{1}, b \subseteq \alpha_{2}$ and $c \subseteq \alpha_{3}$, then

$$
\begin{aligned}
& J\left(h ; \alpha_{1}, \alpha_{2}, \alpha_{3}, a b c\right)=I_{h}\left(\alpha_{2} ; c \mid \alpha_{1} b\right)+I_{h}\left(\alpha_{3} ; b \mid \alpha_{1}\right) \\
& +I_{h}\left(\alpha_{3} ; a \mid \alpha_{2} c\right)+I_{h}\left(\alpha_{1} ; \alpha_{2} \mid \alpha_{3} a b\right)+h\left(c \mid \alpha_{2}\right) .
\end{aligned}
$$

Consequently, if one of the subsets in the Ingleton term $J\left(h ; \alpha_{1}, \alpha_{2}, \alpha_{3}, \alpha_{4}\right)$ is contained in the union of the other three subsets, then the corresponding Ingleton inequality is implied by the basic inequalities. In fact, we will prove that the converse is also true in the following theorem.

Theorem 1: An Ingleton inequality $J\left(h ; \alpha_{1}, \alpha_{2}, \alpha_{3}, \alpha_{4}\right) \geq$ 0 is implied by the basic inequalities if and only if one of the four subsets $\alpha_{1}, \alpha_{2}, \alpha_{3}, \alpha_{4}$ is contained in the union of the other three subsets.

Proof: The if-part follows directly from Property 4 A sketch proof for the converse is given as follows.

Suppose to the contrary that none of the four subsets $\alpha_{1}, \alpha_{2}, \alpha_{3}, \alpha_{4}$ are contained in the union of the remaining three subsets. To prove the converse, it suffices to show that there exists $h^{*} \in \mathcal{F}_{n}$ satisfying every basic inequality but not the Ingleton inequality. To this end, write $\alpha_{i}=\delta_{i} \cup \beta_{i}$, where (1) $\left|\delta_{i}\right|=1$, (2) $\beta_{i} \cap \delta_{i}=\emptyset$ and (3) $\emptyset \neq \delta_{i} \cap \alpha_{j}=\emptyset$ for all $j \neq i$. This term re-writing is possible by assumption. In [12], an entropy function $g$ involving four elements was constructed which satisfies the basic inequalities but not the Ingleton inequality. Since $J\left(h ; \delta_{1}, \delta_{2}, \delta_{3}, \delta_{4}\right)$ involves only four elements, we can easily construct an $h^{*} \in \mathcal{F}_{n}$ that satisfies all the basic inequalities and

$$
\begin{aligned}
J\left(h^{*} ; \alpha_{1}, \alpha_{2}, \alpha_{3}, \alpha_{4}\right) & =J\left(h^{*} ; \delta_{1}, \delta_{2}, \delta_{3}, \delta_{4}\right) \\
& =J\left(g ; \delta_{1}, \delta_{2}, \delta_{3}, \delta_{4}\right)<0 .
\end{aligned}
$$

Hence, $J\left(h^{*} ; \delta_{1}, \delta_{2}, \delta_{3}, \delta_{4}\right)<0$ and the theorem follows.

Define the set of functions with non-negative Ingleton term

$$
\Gamma^{\mathrm{In}} \triangleq\left\{h \in \mathcal{F}_{n}: J\left(h ; \alpha_{1}, \alpha_{2}, \alpha_{3}, \alpha_{4}\right) \geq 0, \forall \alpha_{i} \subseteq \mathcal{N}\right\} .
$$


It is clear that $\Gamma^{\mathrm{In}}$ is a closed and convex cone. To characterize $\Gamma^{\mathrm{In}}$, we first define the following sets of inequalities.

$$
\begin{aligned}
& \Delta_{1}=\{J(h ; i, j, \emptyset, \alpha) \geq 0: i, j \in \mathcal{N}, i \neq j, \mathcal{N} \backslash\{i, j\}\} \\
& \Delta_{2}=\{J(h ; i, i, \emptyset, \mathcal{N} \backslash\{i\}) \geq 0: i \in \mathcal{N}\} .
\end{aligned}
$$

By Property 2, $\Delta_{1}$ and $\Delta_{2}$ together imply all the basic inequalities.

Proposition 2: Let $\alpha_{1}, \alpha_{2}, \alpha_{3}, \alpha_{4}$ be any four subsets of $\mathcal{N}$. Define $\delta_{1}, \delta_{2}, \delta_{3}, \delta_{4}$ and $\beta$ as follows.

$$
\begin{gathered}
\delta_{i} \triangleq \alpha_{i} \backslash \bigcup_{j \neq i} \alpha_{j} \\
\beta \triangleq \bigcup_{i}\left(\alpha_{i} \backslash \delta_{i}\right) .
\end{gathered}
$$

Then $\beta$ contains elements that appear in at least two subsets $\alpha_{i}$. Then, $J\left(h ; \alpha_{1}, \alpha_{2}, \alpha_{3}, \alpha_{4}\right) \geq J\left(h ; \delta_{1} \beta, \delta_{2} \beta, \delta_{3} \beta, \delta_{4} \beta\right)$.

Proof: From Property 3 , if an element appears in more than two subsets, then the element can be "added" to the remaining subsets without increasing the value of the Ingleton term. The result then follows.

Let $\Delta_{0}$ be the set of Ingleton inequalities of the form $J\left(h ; \delta_{1} \beta, \delta_{2} \beta, \delta_{3} \beta, \delta_{4} \beta\right) \geq 0$ where $\delta_{1}, \delta_{2}, \delta_{3}, \delta_{4}$ and $\beta$ are disjoint subsets and $\delta_{1}, \delta_{2}, \delta_{3}, \delta_{4}$ are nonempty. Furthermore, denote $J\left(h ; \delta_{1} \beta, \delta_{2} \beta, \delta_{3} \beta, \delta_{4} \beta\right)$ as $J\left(h ; \delta_{1}, \delta_{2}, \delta_{3}, \delta_{4} \mid \beta\right)$.

Theorem 2: All Ingleton inequalities are implied by the subset of Ingleton inequalities $\Delta=\Delta_{0} \cup \Delta_{1} \cup \Delta_{2}$

Proof: If one subset $\alpha_{i}$ is contained in the union of the other three subsets, then by Theorem 11, the inequality $J\left(h ; \alpha_{1}, \alpha_{2}, \alpha_{3}, \alpha_{4}\right) \geq 0$ is implied by inequalities in $\Delta_{1} \cup \Delta_{2}$. Otherwise, by Proposition 2, the inequality $J\left(h ; \alpha_{1}, \alpha_{2}, \alpha_{3}, \alpha_{4}\right) \geq 0$ is implied by $J\left(h ; \delta_{1}, \delta_{2}, \delta_{3}, \delta_{4} \mid \beta\right) \geq$ 0 in $\Delta_{0}$ where $\beta, \delta_{1}, \cdots, \delta_{4}$ are defined as in (9) and (10).

Hence, $\Gamma^{\mathrm{In}}$ contains all $h \in \mathcal{F}_{n}$ satisfying all inequalities in $\Delta$. Additionally, the set is full-dimensional, as shown below.

Proposition 3: There exists a function $h^{*} \in \mathcal{F}_{n}$ such that $J\left(h^{*} ; \alpha_{1}, \alpha_{2}, \alpha_{3}, \alpha_{4}\right)>0$ for all nontrivial inequalities (i.e., not always zero) $J\left(h ; \alpha_{1}, \alpha_{2}, \alpha_{3}, \alpha_{4}\right) \geq 0$.

Proof: Let $h^{*}(\alpha) \triangleq 2^{n}\left(1-2^{-|\alpha|}\right)$. The proposition then follows by direct verification.

Corollary 1: Suppose $c_{i} \geq 0$ for all $i \in \mathcal{I}$. Then

$$
\sum_{i \in \mathcal{I}} c_{i} J\left(h ; \alpha_{1}^{i}, \alpha_{2}^{i}, \alpha_{3}^{i}, \alpha_{4}^{i}\right)=0
$$

for all $h \in \mathcal{F}_{n}$ if and only if $c_{i}=0$ for all $i \in \mathcal{I}$.

Proof: In the proof of Proposition 3, we constructed $h$ such that $J\left(h ; \alpha_{1}^{i}, \alpha_{2}^{i}, \alpha_{3}^{i}, \alpha_{4}^{i}\right) \geq 1$ for all $i$. Therefore,

$$
\sum_{i} c_{i} J\left(h ; \alpha_{1}^{i}, \alpha_{2}^{i}, \alpha_{3}^{i}, \alpha_{4}^{i}\right) \geq \sum_{i} c_{i} .
$$

Hence, if $\sum_{i} c_{i} J\left(h ; \alpha_{1}^{i}, \alpha_{2}^{i}, \alpha_{3}^{i}, \alpha_{4}^{i}\right)=0$, then $\sum_{i} c_{i}=0$ or equivalently, $c_{i}=0$ for all $i$.

By Proposition 3, we also have the following lemma.

Lemma 1: Suppose that $\delta_{1}, \delta_{2}, \delta_{3}, \delta_{4}, \beta$ are disjoint. Then

1) $J\left(h ; \delta_{1}, \delta_{2}, \delta_{3}, \delta_{4} \mid \beta\right)=0$ if and only if (1) either $\delta_{1}$ or $\delta_{2}$ are empty, and (2) either $\delta_{3}$ or $\delta_{4}$ are empty.
2) $J\left(h ; \delta_{1}, \delta_{2}, \delta_{3}, \delta_{4} \mid \beta\right)=J\left(h ; \delta_{1}^{\prime}, \delta_{2}^{\prime}, \delta_{3}^{\prime}, \delta_{4}^{\prime} \mid \beta^{\prime}\right)$ if and only if (1) $\beta=\beta^{\prime}$, (2) $\left\{\delta_{1}, \delta_{2}\right\}=\left\{\delta_{1}^{\prime}, \delta_{2}^{\prime}\right\}$ and (3) $\left\{\delta_{3}, \delta_{4}\right\}=$ $\left\{\delta_{3}^{\prime}, \delta_{4}^{\prime}\right\}$.

So far, we have proved that the set of inequalities $\Delta$ implies all Ingleton inequalities and hence characterizes $\Gamma^{\mathrm{In}}$. In the following, we will prove that $\Delta$ is indeed the unique minimal set characterizing $\Gamma^{\mathrm{In}}$.

To obtain the minimal set of Ingleton inequalities, we need to overcome an obstacle - that two different choices of $\left\{\alpha_{i}, i=1, \cdots, 4\right\}$ might give the same Ingleton inequality. Therefore, the "repeated" inequalities must be removed.

Example 1: Suppose that $\alpha_{1}=\{1,5\}, \alpha_{2}=\{2,5\}, \alpha_{3}=$ $\{3,5\}$ and $\alpha_{4}=\{4,5\}$. If $\beta_{i}=\alpha_{i}$ for $i=1,2,3$ and $\beta_{4}=$ $\{4\}$, then $J\left(h ; \alpha_{1}, \cdots, \alpha_{4}\right)=J\left(h ; \beta_{1}, \cdots, \beta_{4}\right)$.

Fortunately, by our choice of $\Delta_{1}$ and $\Delta_{2}$, no two inequalities are the same, and by Lemma 1 , two inequalities are the same if and only the subsets in the Ingleton term are permutations of each other as specified in Lemma 1 . Hence, those repeated inequalities can be easily removed. From now on, we assume that no inequalities in $\Delta$ are the same by removing all these duplications.

Theorem 3: No Ingleton inequality in $\Delta$ can be implied by others in $\Delta$. Consequently, the set of Ingleton inequalities $\Delta$ is the unique minimal set of Ingleton inequalities that characterizes $\Gamma^{\mathrm{In}}$.

Proof: The proof for Theorem 3 when $n \leq 5$ can be obtained by brute-force verification. When $n>5$, we will prove Theorem 3 by considering several cases. The proof is rather lengthy and will be given in the next section.

By direct counting, the size of $\Delta$ can be shown to be

$$
n+\left(\begin{array}{l}
n \\
2
\end{array}\right) 2^{n-2}+\frac{1}{4} 6^{n}-5^{n}+\frac{3}{2} 4^{n}-3^{n}+\frac{1}{4} 2^{n} .
$$

\section{PRoOF OF THEOREM 3}

Suppose to the contrary of Theorem 3 that an inequality $J\left(h ; \alpha_{1}, \alpha_{2}, \alpha_{3}, \alpha_{4}\right) \geq 0$ in $\Delta$ is implied by other inequalities $J\left(h ; \alpha_{1}^{i}, \alpha_{2}^{i}, \alpha_{3}^{i}, \alpha_{4}^{i}\right) \geq 0$ in $\Delta$. By Farkas' Lemma ( [13], p.61), there exists non-negative constants $c_{i}$ such that

$$
\begin{gathered}
J\left(h ; \alpha_{1}, \alpha_{2}, \alpha_{3}, \alpha_{4}\right)=\sum_{i \in \mathcal{I}} c_{i} J\left(h ; \alpha_{1}^{i}, \alpha_{2}^{i}, \alpha_{3}^{i}, \alpha_{4}^{i}\right) \\
=\sum_{i \in \mathcal{I}_{0}} c_{i} J\left(h ; \delta_{1}^{i}, \delta_{2}^{i}, \delta_{3}^{i}, \delta_{4}^{i} \mid \beta^{i}\right) \\
+\sum_{i \in \mathcal{I}_{1}} c_{i} J\left(h ; k^{i}, l^{i}, \emptyset, \mu^{i}\right) \\
+\sum_{i \in \mathcal{I}_{2}} c_{i} J\left(h ; m^{i}, m^{i}, \emptyset, \mathcal{N} \backslash\left\{m^{i}\right\}\right) .
\end{gathered}
$$

To prove Theorem 3, it suffices to show that $c_{i}=0$ for all $i \in \mathcal{I} \triangleq \mathcal{I}_{0} \cup \mathcal{I}_{1} \cup \mathcal{I}_{2}$ and hence $J\left(h ; \alpha_{1}, \alpha_{2}, \alpha_{3}, \alpha_{4}\right)=$ 0 contradicting the fact that $\Delta$ does not contain the trivial inequality $0 \geq 0$. We will prove Theorem 3 by considering three exhaustive cases, Case A, Case B and Case C. 
Case A. $J\left(h ; \alpha_{1}, \alpha_{2}, \alpha_{3}, \alpha_{4}\right) \geq 0$ is in $\Delta_{1}$

In this case, the inequality $J\left(h ; \alpha_{1}, \alpha_{2}, \alpha_{3}, \alpha_{4}\right) \geq 0$ is of the form $J\left(h ; i^{0}, j^{0}, \emptyset, \mu^{0}\right) \geq 0$ for some $i^{0} \neq j^{0} \in \mathcal{N}$ and $\mu \subseteq \mathcal{N} \backslash\left\{i^{0}, j^{0}\right\}$.

Now, consider again the equality (14) and project both sides of it away from $i^{0}$. Then the left hand side becomes zero by Lemma 1 . Hence, we have

$$
\begin{aligned}
0=\sum_{i \in \mathcal{I}_{0}} c_{i} J & \left(h ; \delta_{1}^{i} \backslash i^{0}, \delta_{2}^{i} \backslash i^{0}, \delta_{3}^{i} \backslash i^{0}, \delta_{4}^{i} \backslash i^{0} \mid \beta^{i} \backslash i^{0}\right) \\
& +\sum_{i \in \mathcal{I}_{1}} c_{i} J\left(h ; k^{i} \backslash i^{0}, l^{i} \backslash i^{0}, \emptyset, \mu^{i} \backslash i^{0}\right) \\
& +\sum_{i \in \mathcal{I}_{2}} c_{i} J\left(h ; m^{i} \backslash i^{0}, m^{i} \backslash i^{0}, \emptyset, \mathcal{N} \backslash\left\{m^{i}, i^{0}\right\}\right) .
\end{aligned}
$$

By Corollary 1 ,

$$
J\left(h ; \delta_{1}^{i} \backslash i^{0}, \delta_{2}^{i} \backslash i^{0}, \delta_{3}^{i} \backslash i^{0}, \delta_{4}^{i} \backslash i^{0} \mid \beta^{i} \backslash i^{0}\right)=0
$$

for all $i \in \mathcal{I}_{0}$. Then, by Lemma 1] we have either $\delta_{1}^{i}$ or $\delta_{2}^{i}$ is empty, and either $\delta_{3}^{i}$ or $\delta_{4}^{i}$ is empty. However, this is impossible as $\delta_{1}^{i}, \delta_{2}^{i}, \delta_{3}^{i}, \delta_{4}^{i}$ are disjoint and nonempty. Therefore, $c_{i}=0$ for all $i \in \mathcal{I}_{0}$. Consequently,

$$
\begin{aligned}
J\left(h ; i^{0}, j^{0}, \emptyset, \mu\right)= & \sum_{i \in I_{1}} c_{i} J\left(h ; k^{i}, l^{i}, \emptyset, \mu^{i}\right) \\
& +\sum_{i \in I_{2}} c_{i} J\left(h ; m^{i}, m^{i}, \emptyset, \mathcal{N} \backslash\left\{m^{i}\right\}\right) .
\end{aligned}
$$

It is known that the elemental basic inequalities $\Delta_{1} \cup \Delta_{2}$ are not redundant [5]. The theorem is thus proved in this case.

Case B. $J\left(h ; \alpha_{1}, \alpha_{2}, \alpha_{3}, \alpha_{4}\right) \geq 0$ is in $\Delta_{2}$

In this case, we can write the inequality as $J\left(h ; i^{0}, i^{0}, \emptyset, \mathcal{N} \backslash\left\{i^{0}\right\}\right) \geq 0$ for some $i^{0} \in \mathcal{N}$. Again, we can project both sides of the inequality away from $i^{0}$. Using the same argument, we can conclude that $c_{i}=0$ for $i \in \mathcal{I}_{0}$. Then the theorem again follows from that $\Delta_{1} \cup \Delta_{2}$ is not redundant.

Case C. $J\left(h ; \alpha_{1}, \alpha_{2}, \alpha_{3}, \alpha_{4}\right) \geq 0$ is in $\Delta_{0}$

In this case, $J\left(h ; \alpha_{1}, \alpha_{2}, \alpha_{3}, \alpha_{4}\right) \geq 0$ can be rewritten in the form $J\left(h ; \delta_{1}, \delta_{2}, \delta_{3}, \delta_{4} \mid \beta\right) \geq 0$ for some disjoint subsets $\delta_{1}, \delta_{2}, \delta_{3}, \delta_{4}, \beta$ such that $\delta_{i}$ are all nonempty. Again, assume that $J\left(h ; \delta_{1}, \delta_{2}, \delta_{3}, \delta_{4} \mid \beta\right)$ can be written as a linear combination of other Ingleton inequalities as in (14).

First, we will show that $c_{i}=0$ for all $i \in \mathcal{I}_{2}$. Let $h^{*}$ be the entropy function for random variables $\left\{X_{1}, \ldots, X_{n}\right\}$ such that $h^{*}(\alpha)=H\left(X_{i}: i \in \alpha\right)=|\alpha|$. Then, it is straightforward to prove that

$$
\begin{aligned}
J\left(h^{*} ; \delta_{1}, \delta_{2}, \delta_{3}, \delta_{4} \mid \beta\right) & =0, \\
J\left(h^{*} ; \delta_{1}^{i}, \delta_{2}^{i}, \delta_{3}^{i}, \delta_{4}^{i} \mid \beta^{i}\right) & =0 \\
J\left(h^{*} ; k^{i}, l^{i}, \emptyset, \mu^{i}\right) & =0 .
\end{aligned}
$$

Substituting back into (14), we have

$$
0=\sum_{i \in I_{2}} c_{i}
$$

Consequently, $c_{i}=0$ for all $i \in \mathcal{I}_{2}$ and hence

$$
\begin{array}{r}
J\left(h ; \delta_{1}, \delta_{2}, \delta_{3}, \delta_{4} \mid \beta\right)=\sum_{i \in \mathcal{I}_{0}} c_{i} J\left(h ; \delta_{1}^{i}, \delta_{2}^{i}, \delta_{3}^{i}, \delta_{4}^{i} \mid \beta^{i}\right) \\
+\sum_{i \in \mathcal{I}_{1}} c_{i} J\left(h ; k^{i}, l^{i}, \emptyset, \mu^{i}\right) .
\end{array}
$$

Our second task is to show that $c_{i}=0$ for all $i \in \mathcal{I}_{1}$. Again, we will use a similar projection trick. Consider any $i^{0} \in \mathcal{I}_{1}$ and the corresponding inequality

$$
J\left(h ; k^{i_{0}}, l^{i_{0}}, \emptyset, \mu^{i_{0}}\right) \geq 0 .
$$

We can project both sides of (15) onto $k^{i_{0}}$ and $l^{i_{0}}$. Clearly, the right hand side contains the term $c_{i_{0}} J\left(h ; k^{i_{0}}, l^{i_{0}}, \emptyset, \emptyset\right)$. Thus, the left hand side after projection cannot be zero. As a result, (1) $\left\{k^{i_{0}}, l^{i_{0}}\right\} \cap\left(\delta_{1} \delta_{2}\right)$ and $\left\{k^{i_{0}}, l^{i_{0}}\right\} \cap\left(\delta_{3} \delta_{4}\right)$ are nonempty, and (2) $k^{i_{0}}$ and $l^{i_{0}}$ are not in the same subset.

Therefore, we may assume without loss of generality that $k^{i_{0}} \in \delta_{1}$ and that $l^{i_{0}} \in \delta_{3}$. Since $\delta_{2}$ and $\delta_{4}$ are nonempty, we can pick $a \in \delta_{2}$ and $b \in \delta_{4}$. Then we can project (15) onto $\left\{k^{i_{0}}, l^{i_{0}}, a, b\right\}$. After projection, the left hand side becomes

$$
J\left(h ; k^{i_{0}}, a, l^{i_{0}}, b\right)
$$

and the right hand side is a summation of several Ingleton inequalities (involving at most four variables) including $c_{i_{0}} J\left(h ; k^{i_{0}}, l^{i_{0}}, \emptyset, \mu^{i}\right)$. As Theorem 3 holds when $n=4$, we have $c_{i_{0}}=0$. Repeating the same argument for all $i \in \mathcal{I}_{1}$, we prove that $c_{i}=0$ for all $i \in \mathcal{I}_{1}$.

Now (15) can be rewritten as

$$
J\left(h ; \delta_{1}, \delta_{2}, \delta_{3}, \delta_{4} \mid \beta\right)=\sum_{i \in \mathcal{I}_{0}} c_{i} J\left(h ; \delta_{1}^{i} \beta^{i}, \delta_{2}^{i} \beta^{i}, \delta_{3}^{i} \beta^{i}, \delta_{4}^{i} \beta^{i}\right)
$$

Assume that $c_{i}>0$ for all $i \in \mathcal{I}_{0}$ in $(16)$. Now, to prove Theorem 3, it suffices to prove the following statement.

Proposition 4 (Induction Hypothesis $\mathcal{H}(n)$ ): Let $n$ be the number of set elements involved in the left hand side of the expression in (16). Suppose that the equality (16) holds. Then

$$
J\left(h ; \delta_{1}, \delta_{2}, \delta_{3}, \delta_{4} \mid \beta\right)=J\left(h ; \delta_{1}^{i}, \delta_{2}^{i}, \delta_{3}^{i}, \delta_{4}^{i} \mid \beta^{i}\right) .
$$

We have verified cases up to $n \leq 5$. The case when $n \geq 6$ will be proved by induction. To this end, we first prove the claim that any element appearing in the right hand side of 16 must also appear in the left hand side.

Suppose to the contrary that (1) the equality (16) holds and (2) there exists an element $a$ appearing only on the right hand side. Further suppose that $a \in \delta_{j}^{i_{0}}$ for some $i^{0} \in \mathcal{I}_{0}$. Then it is easy to find another element $b$ such that after projection onto $\{a, b\}$, the right hand side of (16) has a term $I_{h}(a, b)$. However, the left hand side of (16) can be shown to be zero, contradicting to Corollary 1 .

On the other hand, if $a \in \beta^{i_{0}}$, then we can pick elements $b \in \delta_{1}^{i_{0}}$ and $c \in \delta_{2}^{i_{0}}$. Projecting both sides of (16) onto $\{a, b, c\}$, the left hand side is either zero or $I_{h}(b ; c)$, while the right hand side of (16) is nonzero as it contains a term $c_{i_{0}} I_{h}(b ; c \mid a)$. Contradiction occurs and hence all elements appearing in the right hand side will also appear in the left hand side. 
Suppose that the induction hypothesis $\mathcal{H}(n)$ holds. We now aim to prove that $\mathcal{H}(n+1)$ also holds. Suppose (16) involves at most $n+1$ set elements where $n \geq 5$. We consider two sub-cases, C.1 and C.2.

Case C.1. $|\beta| \geq 2$ : Let $a, b \in \beta$ and $a \neq b$. Projecting both sides of (16) away from $a$. Then (16) becomes

$$
\begin{aligned}
& J\left(h ; \delta_{1}, \delta_{2}, \delta_{3}, \delta_{4} \mid \beta \backslash a\right) \\
& =\sum_{i \in \mathcal{I}_{0}} c_{i} J\left(h ; \delta_{1}^{i} \backslash a, \delta_{2}^{i} \backslash a, \delta_{3}^{i} \backslash a, \delta_{4}^{i} \backslash a \mid \beta^{i} \backslash a\right) .
\end{aligned}
$$

As (17) involves only $n$ variables, applying the induction hypothesis, we have $\beta_{i}=\beta \backslash a$ or $\beta_{i}=\beta$. Similarly, we can prove that $\beta_{i}=\beta \backslash b$ or $\beta_{i}=\beta$. Consequently, $\beta_{i}=\beta$ for all $i \in \mathcal{I}_{0}$. Again, from (17),

$$
J\left(h ; \delta_{1}, \delta_{2}, \delta_{3}, \delta_{4} \mid \beta \backslash a\right)=J\left(h ; \delta_{1}^{i} \backslash a, \delta_{2}^{i} \backslash a, \delta_{3}^{i} \backslash a, \delta_{4}^{i} \backslash a \mid \beta^{i}\right) .
$$

By Lemma 1 and the induction hypothesis, we have $\left\{\delta_{1}, \delta_{2}\right\}=$ $\left\{\delta_{1}^{i}, \delta_{2}^{i}\right\}$ and $\left\{\delta_{3}, \delta_{4}\right\}=\left\{\delta_{3}^{i}, \delta_{4}^{i}\right\}$. The hypothesis $\mathcal{H}(n+1)$ then holds.

Case C.2. $|\beta| \leq 1$ : Since $n+1 \geq 6$ and $|\beta| \leq 1$, there exists distinct $a$ and $b$ in a subset $\delta_{i}$ for some $i$. Assume without loss of generality that $i=1$. Projecting (16) away from $a$,

$$
\begin{array}{r}
J\left(h ; \delta_{1} \backslash a, \delta_{2}, \delta_{3}, \delta_{4} \mid \beta\right) \\
=\sum_{i \in \mathcal{I}_{0}} c_{i} J\left(h ; \delta_{1}^{i} \backslash a, \delta_{2}^{i} \backslash a, \delta_{3}^{i} \backslash a, \delta_{4}^{i} \backslash a \mid \beta^{i} \backslash a\right) .
\end{array}
$$

By Lemma 1 and the induction hypothesis, $\beta^{i}=\beta$ or $\beta^{i}=$ $\beta \cup\{a\}$. Similarly, by projecting (16) away from $b$, we have $\beta^{i}=\beta$ or $\beta^{i}=\beta \cup\{b\}$. Consequently, $\beta^{i}=\beta$ for all $i \in \mathcal{I}_{0}$. Thus (17) becomes

$$
J\left(h ; \delta_{1}, \delta_{2}, \delta_{3}, \delta_{4} \mid \beta\right)=\sum_{i \in \mathcal{I}_{0}} c_{i} J\left(h ; \delta_{1}^{i}, \delta_{2}^{i}, \delta_{3}^{i}, \delta_{4}^{i} \mid \beta\right) .
$$

and hence after projection away from $a$,

$$
\begin{aligned}
J\left(h ; \delta_{1} \backslash a, \delta_{2}, \delta_{3}, \delta_{4} \mid \beta\right) & \\
& =\sum_{i \in \mathcal{I}_{0}} c_{i} J\left(h ; \delta_{1}^{i} \backslash a, \delta_{2}^{i} \backslash a, \delta_{3}^{i} \backslash a, \delta_{4}^{i} \backslash a \mid \beta\right) .
\end{aligned}
$$

Similarly, projecting (16) away from $b$, we have

$$
\begin{aligned}
J\left(h ; \delta_{1} \backslash b, \delta_{2},\right. & \left.\delta_{3}, \delta_{4} \mid \beta\right) \\
& =\sum_{i \in \mathcal{I}_{0}} c_{i} J\left(h ; \delta_{1}^{i} \backslash b, \delta_{2}^{i} \backslash b, \delta_{3}^{i} \backslash b, \delta_{4}^{i} \backslash b \mid \beta\right) .
\end{aligned}
$$

By (18) and (19), we can then prove that $\left\{\delta_{1}, \delta_{2}\right\}=\left\{\delta_{1}^{i}, \delta_{2}^{i}\right\}$ and $\left\{\delta_{3}, \delta_{4}\right\}=\left\{\delta_{3}^{i}, \delta_{4}^{i}\right\}$. The hypothesis $\mathcal{H}(n+1)$ then holds.

Combining the two cases, we can thus conclude that the set of inequalities in $\Delta$ is not redundant. Since $\Gamma^{\text {In }}$ is fulldimensional, $\Delta$ is indeed the unique, non-redundant set of Ingleton inequalities characterizing $\Gamma^{\mathrm{In}},[13]$, p.64.

\section{CONClusion}

We have identified the unique minimal characterization for the set of polymatroids satisfying all Ingleton inequalities. Knowing this set can greatly simplify computation of IngletonLP bounds for the multicast capacity of linear network codes. Compared to naïve enumeration of all Ingleton inequalities, approximately of the order $16^{n}$, the actual number of necessary inequalities has size approximately of the order $6^{n} / 4-5^{n}$. The complexity reduction is significant, in particular for large $n$.

\section{ACKNOWLEDGEMENT}

This work was supported by the Australian Government under ARC grant DP0557310. This work was performed while L. Guillé was visiting the University of South Autralia.

\section{REFERENCES}

[1] R. Ahlswede, N. Cai, S.-Y. R. Li, and R. W. Yeung, "Network information flow," IEEE Trans. Inform. Theory, vol. 46, no. 4, pp. 1204-1216, July 2000.

[2] S.-Y. R. Li, R. Yeung, and N. Cai, "Linear network coding," IEEE Trans. Inform. Theory, vol. 49, no. 2, pp. 371-381, Feb. 2003.

[3] R. Dougherty, C. Freiling, and K. Zeger, "Insufficiency of linear coding in network information flow," IEEE Trans. Inform. Theory, vol. 51, no. 8 , pp. 2745-2759, Aug. 2005.

[4] L. Song, R. Yeung, and N. Cai, "Zero-error network coding for acyclic networks," IEEE Trans. Inform. Theory, vol. 49, no. 12, pp. 3129-3139, Dec. 2003.

[5] R. Yeung, A First Course in Information Theory. Kluwer Academic/Plenum Publisher, 2002.

[6] R. W. Yeung, S.-Y. R. Li, N. Cai, and Z. Zhang, Network Coding Theory, ser. Foundations and Trends in Communications and Information Theory. Now Publishers, 2006.

[7] X.Yan, R. W. Yeung, and Z. Zhang, "The capacity region for multisource multi-sink network coding," in IEEE Int. Symp. Inform. Theory, 2007.

[8] F. Matus, "Infinitely many information inequalities," 2007.

[9] T. Chan and A. Grant, "Dualities between entropy functions and network codes," submitted to IEEE Trans. Inform. Theory. [Online]. Available: http://arxiv.org/abs/0708.4328v1

[10] R. Yeung, "A framework for linear information inequalities," IEEE Trans. Inform. Theory, vol. 43, no. 6, pp. 1924-1934, Nov. 1997.

[11] F. Matus, "Adhesivity of polymatroids," Discrete Math., 2007.

[12] Z. Zhang and R. W. Yeung, "On the characterization of entropy function via information inequalities," IEEE Trans. Inform. Theory, vol. 44, pp. pp. 1440-1452, 1998

[13] A. Schrijver, Combinatorial Optimization: Polyhedra and Efficiency (Algorithms and Combinatorics). Springer-Verlag, Berlin, 2003. 